(C) Elsevier/INRA

\title{
Mesures des déformations résiduelles de croissance à la surface des arbres, en relation avec leur morphologie. Observations sur différentes espèces
}

\author{
M Fournier ${ }^{1}$, B Chanson 1, B Thibaut 2, D Guitard ${ }^{1}$ \\ 1 INRA, centre de recherches forestières, laboratoire de rhéologie du bois de Bordeaux, \\ UMR CO23 du CNRS. \\ domaine de I'Hermitage, Pierroton, 33610 Cestas; \\ 2 Université de Montpellier II, laboratoire de mécanique et de génie civil, URA 1214 du CNRS, \\ place Eugène-Bataillon, 34095 Montpellier Cedex 5, France
}

(Reçu le 9 octobre, accepté le 12 février 1993)

\begin{abstract}
Résumé - Le bois des arbres sur pied est soumis à des efforts internes permanents, appelés contraintes "de croissance", résultant de déformations de maturation non exprimées lors de la différenciation du bois dans l'arbre. Le but de notre programme de recherches «Architecture, Structure, Mécanique de l'arbre" est de mettre en évidence les relations entre ces déformations de maturation, la structure anatomique du bois et la morphogenèse de l'arbre, dans l'objectif de modéliser les contraintes supportées par le bois et d'estimer les risques de fentes et de déformations à la transformation. Une synthèse des résultats en cours concernant les déformations longitudinales résiduelles mesurées à la surface, qui estiment les déformations de maturation, est présentée à propos de plusieurs individus ou populations. Deux méthodes différentes de mesure sont utilisées et comparées. Á l'échelle de l'individu, les déformations mesurées varient avec la hauteur et avec la position angulaire sur la circonférence ; ces observations sont analysées en relation avec la morphogenèse de l'arbre : proximité de branches, redressements des axes. De fait, les valeurs élevées de déformations ne sont jamais homogènes mais concentrées sur de petits secteurs angulaires. Ceci permet à l'arbre de se réorienter puisqu'une face "tire" ou "pousse" l'autre. Ceci entraîne que la distribution des valeurs mesurées n'est pas normale. La "trainée" plus ou moins importante de valeurs élevées en tension (chez les feuillus) ou en compression (chez le résineux) est reliée à la formation de bois de réaction. La variabilité entre populations d'arbres ne réside donc pas dans des valeurs moyennes différentes mais dans la structure de la traînée, c'est-à-dire dans la fréquence, l'amplitude et la largeur des pics angulaires de déformation. Ceci conduit à étudier les contraintes de croissance en corrélation étroite avec la régulation de l'inclinaison et les variations de courbures des axes lignifiés en croissance. Pour finir, des distributions angulaires surprenantes de déformations résiduelles, montrant 2 pics opposés, observées sur Dichostemma sp, Saccoglotis gabonensis, Eperua falcata ainsi que Castanea sativa, sont discutées. Ces distributions peuvent être reliées à l'architecture de l'arbre: dans ces arbres sympodiaux (ie où le tronc est construit par empilement successifs d'axes issus de bourgeons axillaires), les pics de déformations proviennent des différents axes (le tronc actuel et l'ancien devenu branche) avec une sorte de compétition.
\end{abstract}

contraintes de croissance / méthodes de mesure / architecture de l'arbre 
Summary - Measurements of residual growth strains at the stem surface. Observations on different species. The wood in standing trees undergoes internal stress during the entire life of the tree. This stress, commonly named 'growth stress', originates in maturation strains and is impeded by the mass of the entire trunk. Released strain at the stem surface (ie strain on a small piece of wood isolated from the stem by cutting grooves, drilling holes etc) measures maturation strain. Our research program on 'Tree architecture, anatomy and mechanics' aims at: i) understanding the biological control of maturation strain with regard to tree morphogenesis (branching patterns, crown form, stem and shoot positions in relation to vertical direction); ii) qualifying correlations between maturation strain and the anatomical features of the wood and other wood characteristics (stiffness, shrinkage, hygrothermal recovery); iii) modelizing and qualifying the stress in the entire tree that results from the cumulative maturation of successive layers during cambial growth, in order to understand cracks and strains when the wood is processed. This paper focuses on results concerning longitudinal maturation strain at the stem periphery measured on different species. Two methods have been used: i) the single hole method with a special sensor designed in the Centre Technique Forestier Tropical; and ii) measurements of strain due to 2 grooves sawn above and below a classical electric sensor. Comparisons between the 2 methods show quite good, but not perfect, agreements in beech and eucalyptus but not in chesnut. These results are discussed from the sensor dimensions, the principles of the method and the anatomical and mechanical properties of wood. Measurements on one tree show important variations with height and angular position, which are correlated to tree morphogenesis (proximity of branches and righting movements of stems). High strain values are never homogeneous in the tree but concentrated in small angular sectors. This angular asymmetry of maturation strain is obviously related to stem bending movement as one side of the stem 'pulls' or 'pushes' the other. Furthermore, histograms of values measured on Pinus pinaster, clones of Eucalyptus (PF1 1.45, UAIC-CTFT, Congo) and poplar (Populus euramericana cv /214), Castanea sativa, Fagus sylvatica and Eperua falcata show that the distribution of strains is not Gaussian. The long tail of tensile (in hardwoods) or compressive (in softwood) values correlates with the formation of reaction wood (compression or tension). The main difference between populations is not the mean value (out of the tail, in 'normal standard wood') but the width and maximum of the tail. Hence, to study variability of growth stress in a population of trees, we must study the frequency and the magnitude of 'peaks of high maturation strains' within trees, rather than mean strains. Therefore, growth stress should be analyzed in correlation with the regulation of the form of the tree and particularly in correlation with the kinetics of stem movement (changes of curvature and lean). Finally, uncommon patterns of release strain with 2 opposite angular peaks have been observed in some tropical trees as Dichostemma $s p$. Saccoglotis gabonensis, Eperua falcata and Castanea sativa. These patterns can be related to the tree architecture. In sympodial trees (ie trees in which the trunk is built by stacks of branches as stems formed from axillary buds take over from the former leader), peaks of high maturation strains seem to be induced by the different axes (the present leader and the former) and thus, in a cross-section, 2 flows of highly strained wood can be observed. A functional explanation of such patterns is not obvious.

\section{growth strain / measurement technique / tree architecture}

\section{INTRODUCTION}

On rappelle que le bois dans l'arbre est soumis à des efforts d'origine interne induits pendant la différenciation cellulaire (de l'ordre de $10 \mathrm{MPa}=100 \mathrm{~kg} / \mathrm{cm}^{2}$ dans la direction longitudinale). La surface de l'arbre, faite de bois très jeune, n'a subi que les effets de sa propre maturation. L'estimation de son état de contraintes, c'est-à-dire de la tendance à se déformer d'un volume élémentaire de bois de cette surface lorsqu'on l'isole du reste du tronc, est donc facilement interprétable en termes de "déformation de maturation" (Fournier, 1989). Cette déformation de maturation est une grandeur rhéologique que l'on peut espérer relier à la structure anatomique du bois. D'un point de 
vue «biologique», elle est une grandeur de la dynamique de la croissance secondaire qui traduit l'état actuel (ou du moins immédiatement passé) de la différenciation du bois. Si l'on admet que les différents méristèmes et processus de différenciation fonctionnent en interrelation et sont influencés par des facteurs endogènes ou externes, on peut donc espérer relier cette grandeur à d'autres états (morphologique ou architectural ?, phénologique?, génétique?) de l'arbre et de son milieu. Notamment, la dissymétrie sur la circonférence des déformations de maturation traduit les réorientations en cours (ou stabilisations) par la croissance secondaire de l'arbre (Fournier, 1989).

Cette déformation de surface à différentes étapes de croissance (ie fonction du diamètre ou de l'âge) est la base de la mise au point de modélisation de l'état des contraintes dans le tronc. En effet, l'état des contraintes dans l'ensemble de la grume dépend de toute l'histoire de l'arbre. L'objectif appliqué immédiat de l'étude de ces déformations est l'analyse mécanique des voilements et tuilages des pièces sciées ainsi que des ruptures provoquées par la modification du champ initial des contraintes lors de l'abattage ou du sciage. Le problème des ruptures (fentes d'abattage...) requiert la prise en compte des propriétés aux états limites du matériau (enveloppes de rupture, fissilité). Dans les 2 cas, il faut ajouter à l'étude du champ des contraintes initial dans l'arbre (à la surface et à l'intérieur) celle des effets du procédé de libération lui-même qui sont susceptibles d'être très sensibles : pour un même arbre, des techniques d'abattage différentes (profondeur d'entaille, hauteur de charnière...) risquent de provoquer des fentes très différentes. Bien que les déformations de maturation de surface étudiées interviennent dans le problème mécanique de l'apparition de fentes dans les grumes et billes, il faut donc conserver à l'esprit que les corrélations (entre par exemple valeur maximale mesurée et dimensions des fentes), si elles risquent d'exister, n'ont aucune raison d'être immédiates et demandent une analyse ultérieure purement mécanique du procédé de découpe. Notre programme de recherche "Architecture, structure, mécanique de l'arbre» (Thibaut, 1989) se donne pour objectif général :

i) d'expliciter le contrôle biologique de ces déformations de maturation pendant la morphogenèse de l'arbre en les mettant en relation avec le modèle architectural de l'espèce et des dynamiques de croissance particulières dans certains environnements (relais traumatiques à la suite à la mort de lapex, édification de la cime et développement de branches maîtresses, inclinaison anormale des axes provoquée par un déchaussement...) (Loup et al, 1991 ; Chanson, 1993) ;

ii) de qualifier les relations entre les déformations de maturation et les caractéristiques anatomiques du bois (Yamamoto et Okuyama, 1988), ainsi qu'avec d'autres propriétés physico-mécaniques : retraits, recouvrance hygrothermique ;

iii) de modéliser et de qualifier les champs de contraintes dans l'ensemble de la tige qui sont le résultat de la formation et de la maturation de couches périphériques successives pendant la croissance secondaire (Archer, 1986a ; Fournier et al, 1991);

iv) de modéliser et de qualifier les déformations et ruptures des débits à partir de ce champ de contrainte initial (Serres et Zarka, 1977 ; Archer, 1986a ; Gérard et al, 1991) et des propriétés mécaniques usuelles (rigidités, résistances, fissilités) du bois vert.

Le présent article a pour objectif d'énoncer quelques points essentiels concernant la mesure et la variabilité des déformations de maturation, ainsi que quelques résultats peu communs et troublants concernant le premier volet du programme (relations entre déformations de maturation et morphogenèse de l'arbre). 


\section{MATÉRIELS ET MÉTHODES}

Le matériel végétal auquel il sera fait référence dans le reste de l'étude concerne:

- une population de 12 hêtres (peuplement régulier de 50 ans) (Chanson et al, 1992) ;

- une population de 14 châtaigniers limousins traités en taillis (étude IDF à paraître);

- une population de 14 peupliers (clone 1 214) (Castéra et al, 1994) ;

- une population de 16 eucalyptus (clone 1.45 de l'Unité d'afforestation industrielle du Congo [UAIC]) (Baillères et al, 1992) ;

-2 pins maritimes de 25 ans (Loup ef al, 1991) ;

(Dans la mesure où ces populations font l'objet d'études particulières, le lecteur est renvoyé aux publications ci-dessus pour de plus amples informations. Le présent article se bornera à une comparaison entre populations sans entrer dans l'analyse de leur variabilité propre.)

- 2 Eperua falcata de forêt équatoriale primaire guyanaise (arbres de bord de piste, proches du camp de la Piste-Saint-Élie) ;

- un Dichostemma sp et un Sacoglottis gabonensis, de la forêt équatoriale camerounaise, observés lors de l'opération “Canopées" 1991).

Le premier problème est la mesure des déformations de maturation à la surface des arbres ! Jusqu'à ce jour, cette mesure n'a fait l'objet d'aucune norme, chaque chercheur mettant au point sa propre méthode ( $c f$ synthèse dans Archer, 1986a ; Fournier, 1989), certains auteurs courageux tentant une synthèse des résultats proposés par les uns et les autres (Kübler, 1987).

Notre équipe a utilisé 2 méthodes pour l'estimation de la déformation longitudinale de maturation (notée désormais $\alpha L$ ).

La première méthode (dénommée désormais "méthode CTFT»), qui repose sur la méthode dite du trou unique (Archer, 1986b), utilise un capteur mis au point au Centre technique forestier tropical. Le principe est de mesurer la variation de distance $d$, due au perçage d'un trou central de $20 \mathrm{~mm}$, entre 2 pointes distantes de $4,5 \mathrm{~cm}$ selon le fil du bois et enfoncées, après écorçage (jusqu'au cambium), sur une profondeur de $10 \mathrm{~mm}$. Cet essai mécanique s'appuie sur le principe suivant : à géométrie de capteur fixée (distance entre pointes, diamètre du trou), plus le retrait de maturation $\alpha_{L}$ (responsable de la tension longitudinale du bois à la surface de l'arbre) est grand, plus les pointes s'écartent lors du perçage. Une analyse mécanique rigoureuse montre que la variation mesurée dépend en outre de l'anisotropie élastique du bois dans le plan Iongitudinal-tangentiel et des composantes du champ des contraintes autres que longitudinales, mais que ces paramètres restent peu sensibles. Cette hypothèse n'a toutefois pas été réellement vérifiée pour les bois de tension dont les caractéristiques mécaniques sont à la fois mal connues et vraisemblablement très différentes des bois normaux ; de même l'influence de défauts locaux tels qu'ondulations locales du fil, présence de petits nœeuds est assez mal contrôlée : on essaie donc dans la mesure du possible de se placer dans une zone de bois "propre".

La seconde méthode (dénommée désormais "méthode Wap's") consiste à mesurer, sur une base de mesure de $14 \mathrm{~mm}$, la déformation longitudinale résultant de l'usinage (par sciage annuel), de part et d'autre du capteur de déformation, de 2 entailles situées à $5 \mathrm{~mm}$ des pointes et profondes d'environ $7 \mathrm{~mm}$ (fig 1). Le capteur de déformation est un extensomètre à jauges de contraintes (HBM) fixé à l'arbre par l'intermédiaire de pointes de compas enfoncées sur une profondeur de 1 à $2 \mathrm{~mm}$, et relié à une centrale d'acquisition. À géométrie fixée (base de mesure du capteur, distance des entailles aux pointes et profondeur du sciage), plus le retrait $\alpha_{\mathrm{L}}$ est grand, plus le retrait $\varepsilon_{\mathrm{L}}$ mesuré par le capteur est grand. $A$ priori, cette méthode des 2 rainures sur une base de mesure aussi petite libère entièrement les contraintes de sorte qu' $\alpha_{L}$ serait non seulement proportionnel mais aussi égal à $\varepsilon_{L}$. Cette hypothèse mérite cependant d'être vérifiée. Enfin, cette deuxième méthode donne une information plus locale que la précédente, à la fois sur la surface (base de mesure de $14 \mathrm{~mm}$ ) et sur la profondeur.

Dans le but de comparer les 2 méthodes, nous avons doublé un grand nombre de points de
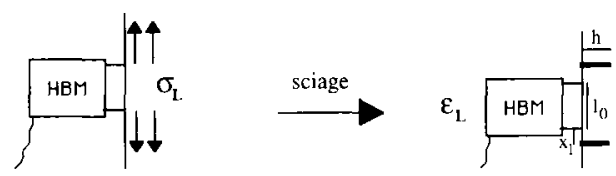

Fig 1. Principe de la mesure des contraintes par la mesure de la déformation provoquée par le sciage de 2 rainures libérant les contraintes longitudinales initiales de tension. Dans notre dispositif expérimental, $\mathrm{I}_{0}=14 \mathrm{~mm}, \mathrm{x}_{1}=5 \mathrm{~mm}$ et $\mathrm{h}=7 \mathrm{~mm}$. 
mesures (2 points «jumeaux» sont situés sur la même génératrice à environ $20 \mathrm{~cm}$ l'un de l'autre).

Enfin, dans le but d'observer la variabilité des déformations à l'échelle de l'individu, un grand nombre de mesures ont parfois été réalisées sur le même arbre. En particulier, 4 à 12 mesures sur une même circonférence sont généralement effectuées.

\section{MÉTROLOGIE}

Les résultats de la comparaison des 2 méthodes sur les hêtres, les châtaigniers et les eucalyptus sont donnés sur les figures 2,3 et 4 . On note que la corrélation est bonne sur le hêtre et l'eucalyptus mais loin d'être excellente, comme s'il s'agissait d'un simple étalonnage !

La modélisation mécanique de la méthode CTFT du trou unique (Archer,

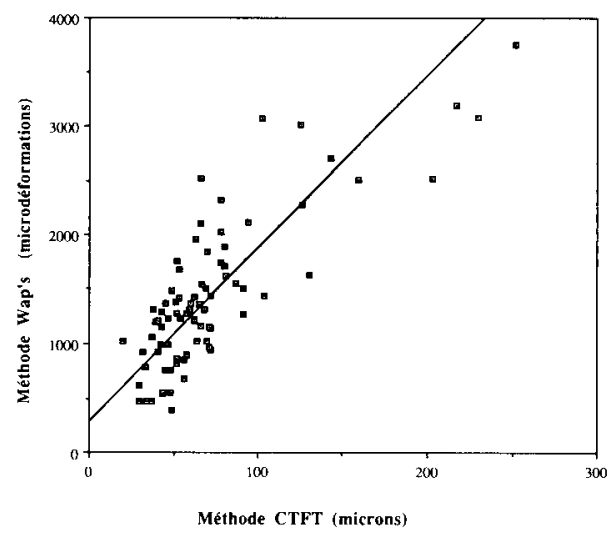

Fig 2. Corrélation observée sur le hêtre entre les 2 méthodes de mesure (en abscisse $x$, la variation de distance en microns mesurée par le capteur CTFT, en ordonnée $y$, le retrait mesuré par la méthode Wap's en microdéformations). La droite représentée, de pente $15,8 \mu \mathrm{def} /$ micron, est l'axe d'inertie du nuage et non l'une des 2 droites de régression $y=f(x)$ (de pente $12,9 \mu$ edf/micron) ou $x=f(y)$ (de pente $1 / 19,4$ micron/ $\mu$ def) puisque rien ne permet d'affirmer qu'une des 2 mesures est connue sans erreur. Le coefficient de corrélation est 0,81 , significatif au seuil de $0,001 \%$ (effectif 78). 1986a, Gérard et al, 1991) donne une relation entre la mesure $\delta$ effectuée en microns et $\alpha \mathrm{L}$ compte tenu de la géométrie du capteur (distance entre points et diamètre du trou), et des rapports d'anisotropie élastique du bois dans le plan LT. Les applications numériques menées pour différents. cas d'anisotropie élastique prévoient que $-\alpha_{\mathrm{L}}=$ $\mathrm{K}_{\text {mod }} \delta$ avec $: K_{\text {mod }}=12,3 \mu \mathrm{def} / \mu \mathrm{m}$ pour le feuillu standard, $10,5 \mu \mathrm{def} / \mu \mathrm{m}$ pour Populus tremuioides, $8,8 \mu \mathrm{def} / \mu \mathrm{m}$ pour Pinus sp, $9,6 \mu \mathrm{def} / \mu \mathrm{m}$ pour Eperua falcata, $11,6 \mu \mathrm{def} / \mu \mathrm{m}$ pour Juglans sp (en utilisant les valeurs de constantes élastiques données par Guitard (1987), corrigées de l'état sec à l'état vert (Fournier, 1989).

Si l'on admet que la méthode Wap's, en libérant totalement les contraintes, donne directement et sans biais $\alpha_{L}$, et que la variance résiduelle par rapport à la droite de régression est le fait d'un effet aléatoire

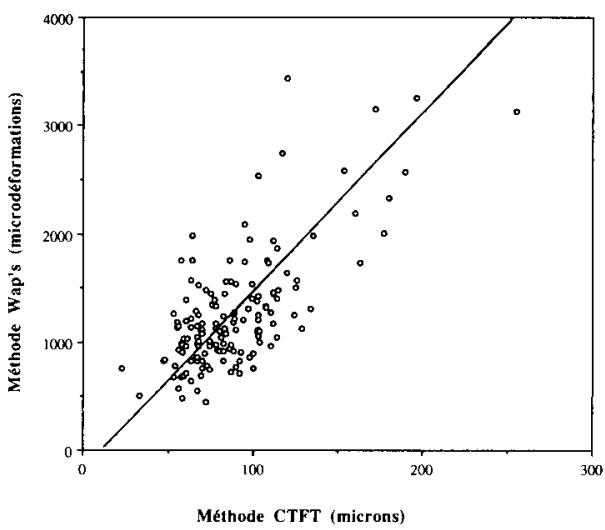

Fig 3. Corrélation observée sur l'eucalyptus (Clone 1,45, UAlC Congo) entre les 2 méthodes de mesure (en abscisse $x$, la variation de distance en microns mesurée par le capteur CTFT ; en ordonnée $\mathrm{y}$, le retrait mesuré par la méthode Wap's en microdéformations). La droite représentée, de pente $16,5 \mu \mathrm{def} /$ micron, est l'axe d'inertie du nuage et non l'une des 2 droites de régression $y=f(x)$ (de pente 11,6 $\mu$ def/micron) ou $x=f(y)$ (de pente $1 / 23,4$ micron/ 1 def) puisque rien ne permet d'affirmer qu'une des 2 mesures est connue sans erreur. Le coefficient de corrélation est 0,71 , significatif au seuil de 0,001\% (effectif 147). 


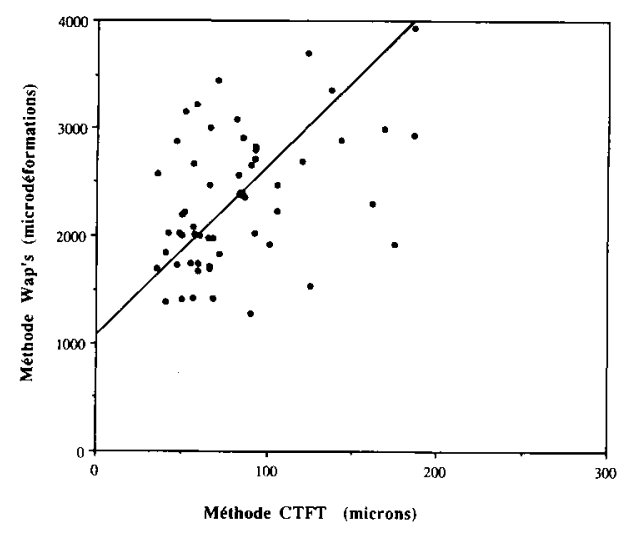

Fig 4. Corrélation observée sur le Chataîgnier entre les 2 méthodes de mesure (en abscisse $\mathrm{x}$, la variation de distance en microns mesurée par le capteur CFTF ; en ordonnée $y$, le retrait mesuré par la méthode Wap's en microdéformations). La droite représentée, de pente $17,8 \mu \mathrm{def} / \mathrm{mic}$ ron, est l'axe d'inertie du nuage et non l'une des 2 droites de régression $y=f(x)$ (de pente $8,7 \mu$ def/micron) ou $x=f(y)$ (de pente 1/36,4 micron/udef) puisque rien ne permet d'affirmer qu'une des 2 mesures est connue sans erreur. Le coefficient de corrélation est 0,49 , significatif au seuil de $0,001 \%$ (effectif 56 ).

(la méthode CTFT homogénéise la mesure sur une plage de bois plus vaste que la méthode Wap's très locale, les 2 couronnes de mesure sont distantes de 10 à $20 \mathrm{~cm}$ ), la pente de la droite de régression $K_{\text {est }}$ devrait être proche du coefficient modélisé $K_{\bmod }$. De fait, les pentes estimées semblent plus fortes mais rien ne permet d'affirmer qu'elles sont significativement différentes. En particulier, les pentes du modèle linéaire "Méthode Wap's $=\mathrm{K}_{\text {est }}{ }^{*} \times$ Méthode CTFT + E" (qui suppose une absence d'erreur aléatoire significative sur la Méthode CTFT prise comme variable explicative de référence), sont $K_{\text {est }}{ }^{*}=12,9 \mu \mathrm{def} / \mu \mathrm{m}$ pour le hêtre, et $\mathrm{K}_{\text {est }}{ }^{\star}=11,6 \mu \mathrm{def} / \mu \mathrm{m}$ pour l'eucalyptus ; I'intervalle de confiance à $95 \%$ sur ces coeffi- cients $\mathrm{K}_{\mathrm{est}}{ }^{*}$ comprend l'intervalle des valeurs modélisées.

Sur le châtaignier, la corrélation est bien plus mauvaise (bien que restant significative à la probabilité 0,001 ). Vu que notre manière de procéder est restée identique, il faut donc incriminer les arbres eux-mêmes (et non les méthodes en général ni les expérimentateurs en particulier !). Le problème pourrait venir de l'hétérogénéité intra-cerne du châtaignier (la zone initiale poreuse était en train de se finir lorsque nous avons fait les mesures en juillet). La méthode Wap's, très locale (comme l'ont montré des résultats antérieurs sur Pinus pinaster) mesurerait les déformations de maturation dans la partie du cerne située à la surface, la méthode CTFT homogénéisant la mesure sur plusieurs cernes. Compte tenu des propriétés très différentes des bois initiaux et finaux, rien d'étonnant à ce que la corrélation soit brouillée! ceci reste cependant une hypothèse à vérifier.

On retiendra qu'en l'état des connaissances il faut être prudent en comparant des valeurs obtenues par des techniques différentes. Le choix d'une méthode (pointes plus ou moins profondes, jauges collées, large ou petite base de mesure...) n'est pas a priori conditionné seulement par des aspects pratiques mais aussi par ce que I'on cherche à obtenir (mesure très locale ou homogénéisée).

\section{VARIATIONS DES DÉFORMATIONS DE MATURATION À L'ÉCHELLE D'UNE POPULATION D'ARBRES : INCIDENCE DES VARIATIONS ANGULAIRES SUR LA CIRCONFÉRENCE}

Les mesures effectuées sont rarement homogènes mais présentent généralement un pic sur la circonférence : pic de tension (retrait $\alpha_{L}$ important) chez les feuillus (fig 5), pic de compression ( $\alpha_{L}$ devient un fort gonflement) chez les conifères (fig 6). Nous 
avons choisi de repérer conventionnellement par la position angulaire $0^{\circ}$ la face inférieure de la tige inclinée. Lorsque le tronc est droit (ce qui est assez rare), $0^{\circ}$ est positionné dans la direction vers laquelle le houppier est excentré. Enfin, lorsque le tronc est droit et le houppier symétrique (ce qui est extrêmement rare), $0^{\circ}$ est une position aléatoire mais repérée par rapport à la morphologie de l'individu (position des branches). De fait, la position des pics de déformations est généralement corrélée avec la face supérieure (sur les feuillus) ou inférieure (sur les conifères) de la tige inclinée. Cette observation a été mentionnée par de nombreux auteurs (Saurat et Guéneau, 1976 (sur le hêtre) ; Archer, 1986a).

À l'échelle d'une population d'arbres (ou de sections droites d'arbres), la différence essentielle entre 2 populations réside dans la fréquence et l'amplitude des pics ou des maxima de tension sur chaque population. Les valeurs "normales» (= hors des pics) ne semblent pas sensiblement différentes. Cela apparaît clairement sur les figures 7 et 8 qui relatent les valeurs mesurées sur la population «peu contrainte» des 14 châ- taigniers et la population "très contrainte» des 14 peupliers (1214). (Les qualificatifs "peu contrainte" et "très contrainte", conformes à la réputation de ces essences, ont été donnés sur un critère indépendant d'observations de fentes à cœur plus ou moins importantes à l'abattage.)

Ce résultat implique que les distributions mesurées ne sont pas normales et qu'elles diffèrent moins par leur valeur la plus fréquente que par l'amplitude de leur traînée (vers les valeurs de tension pour les feuillus, les valeurs en compression pour le conifère), comme le montrent les histogrammes des figures 9 à 14. En outre, cette non normalité des distributions mesurées apparaît quel que soit le nombre d'arbres de l'échantillon puisqu'elle résulte d'une variabilité intra-arbre. Ainsi, les peupliers et les Eperua falcata étudiés apparaissent comme des populations "contraintes" du fait de leur longue traînée dans les valeurs positives, alors que les pins maritimes développent leur traînée dans l'autre sens vers les valeurs négatives, du fait de la présence de bois de compression. Enfin, certaines distributions (Eperua falcata, hêtres, châtaigniers) semblent bimodales, ce qui pourrait
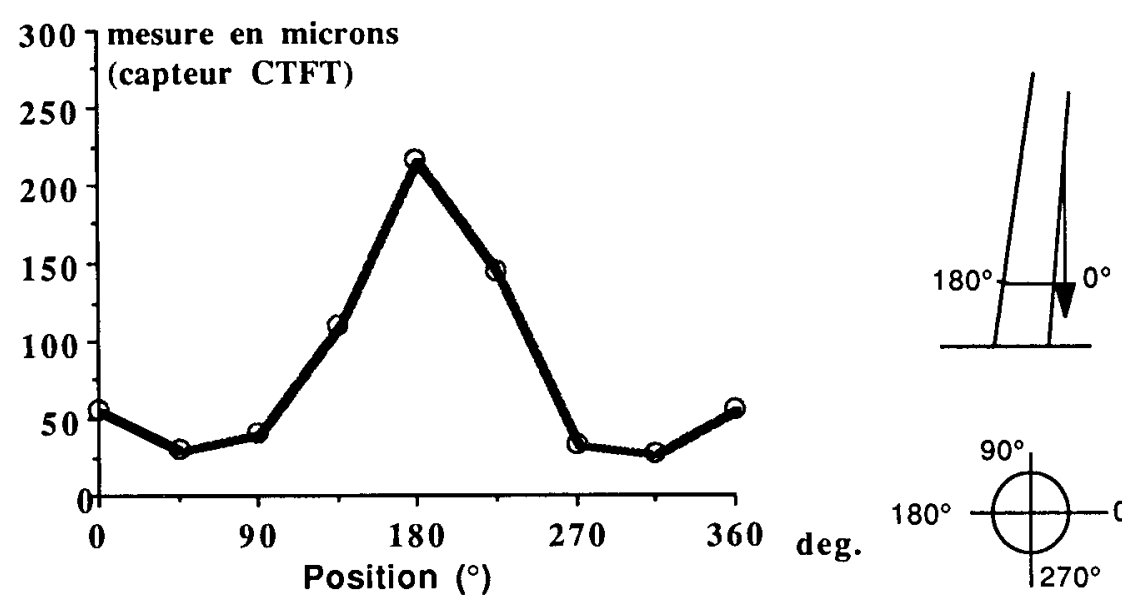

Fig 5. Exemple typique de variation angulaire de déformation de maturation mesurée sur la circonférence d'un arbre feuillu. Les mesures représentées ont été effectuées à 1,30 m sur un Fagus sylvatica. 


\section{Pin maritime 1}

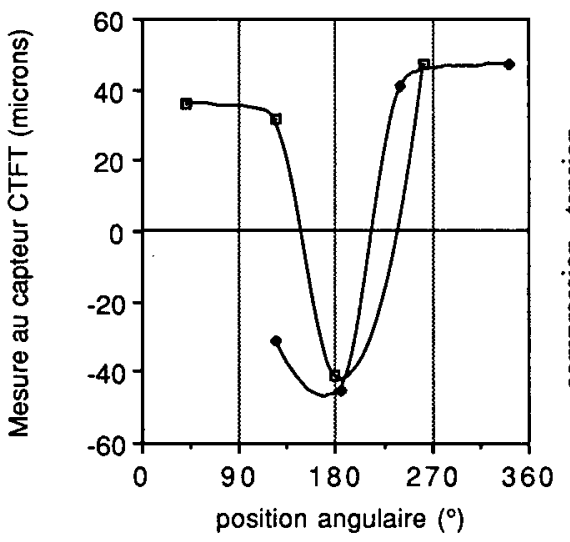

Fig 6. Exemple typique de variation angulaire de déformation de maturation mesurée sur la circonférence d'un conifère en présence de bois de compression autour de la position $180^{\circ}$. Les mesures représentées ont été effectuées sur 2 interverticilles du même Pinus pinaster.

témoigner de la présence d'une certaine discontinuité entre du bois normalement contraint et du bois de tension, alors qu'une traînée plus continue signifierait que la transition bois normal-bois de tension passe par toute sorte de structures intermédiaires. Ces hypothèses devrait être vérifiées ultérieurement par l'observation plus systématique de populations importantes couplée à des observations anatomiques. De fait, compte tenu de I'hétérogénéité des populations analysées (variations intra-arbre sur 2 pins maritimes ou 2 Eperua falcata seulement, arbres clonaux ou non, arbres de plantation, de taillis, de futaie, de forêt naturelle guyanaise...) qui n'ont nullement la prétention de représenter un échantillon significatif de l'espèce étudiée, la comparaison des distributions ne permet pas en l'état l'analyse rigoureuse des effets du génotype ou des conditions de croissance. De telles analyses demandent des échan- tillons plus larges tels que ceux de Saurat et Guéneau (1976) ou Polge (1981) sur la hêtraie française. Toutefois, notre analyse montre que, compte tenu de la variance importante et de la non-normalité des distributions de déformations mesurables sur un seul arbre, il faut également rester prudent lors de l'interprétation des résultats obtenus à partir de peu de mesures par arbre sur de nombreux individus. Notamment, la signification de la "valeur moyenne d'un arbre" estimée par la moyenne de 5 mesures à 1,30 m (Saurat et Guéneau, 1976) doit être discutée.

\section{RELATIONS ENTRE LES PICS DE DÉFORMATION OBSERVÉS ET LA MORPHOLOGIE DE L'ARBRE}

Un pic de déformation de maturation permet à l'axe de se réorienter puisque la face la plus tendue «tire" alors l'autre et courbe ainsi l'ensemble de la tige. II est donc logique d'observer des pics de déformation sur les axes en réorientation et des profils plats sur les axes n'étant pas en cours de réorientation. De plus, les troncs étant généralement des axes orthotropes, les axes en réorientation seront généralement les tiges inclinées. De fait le résultat le plus courant est d'obtenir des pics importants sur les arbres les plus penchés alors que les profils de déformations sont en comparaison très plats sur les arbres les plus droits (figs 15 et 16 ; et Saurat et Guéneau, 1976 ; Archer, 1986a). II n'est cependant pas rare d'obtenir des résultats impossibles à interpréter aussi simplement. II s'agit par exemple de valeurs fortement dissymétriques sur des arbres droits, de variations longitudinales rapides des déformations mesurées ou enfin, de l'apparition de 2 pics de déformations diamétralement opposés. L'interprétation passe alors par l'analyse du fonctionnement de l'arbre, relatif à son architecture et à sa morphologie. Un exposé plus profond de ces concepts et de 


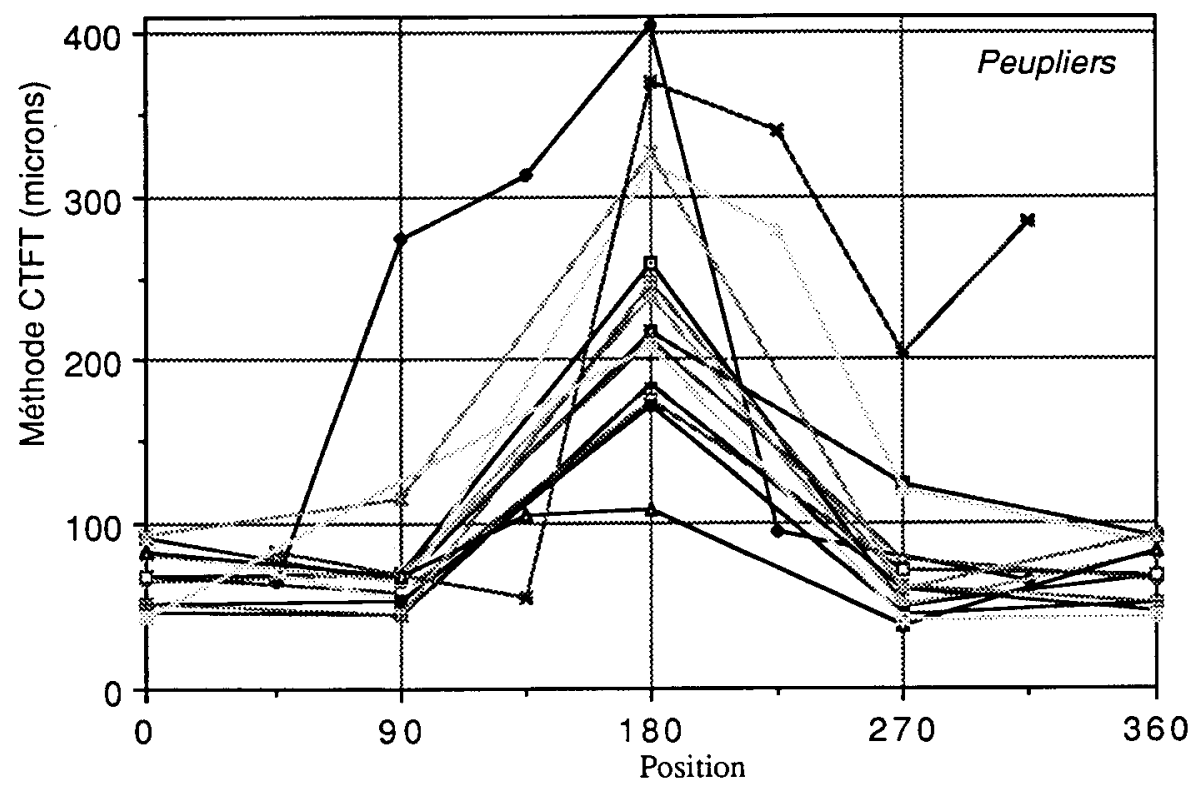

Fig 7. Déformations mesurées sur la circonférence de 14 peupliers (clone 1214).

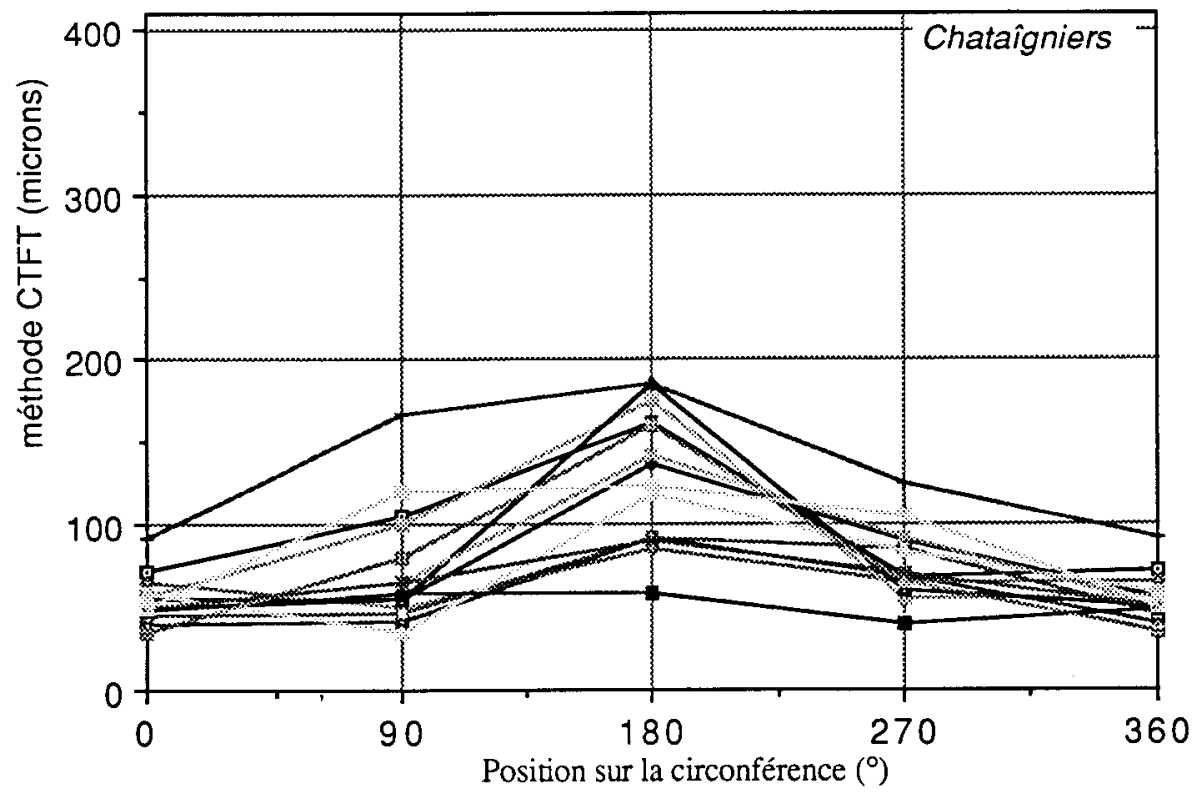

Fig 8. Déformations mesurées sur la circonférence de 14 Châtaigniers (taillis Limousin). 


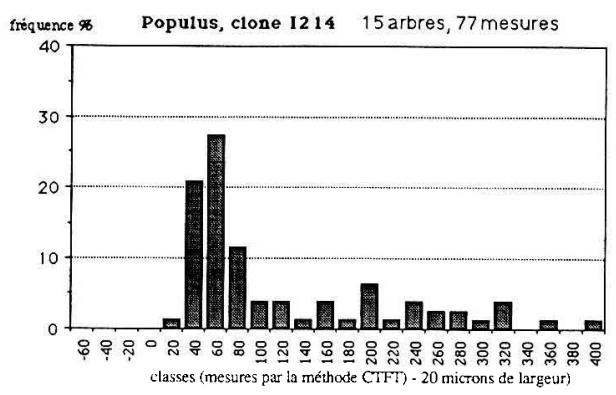

Fig 9. Distribution des déformations mesurées à la surface des Peupliers 1214.

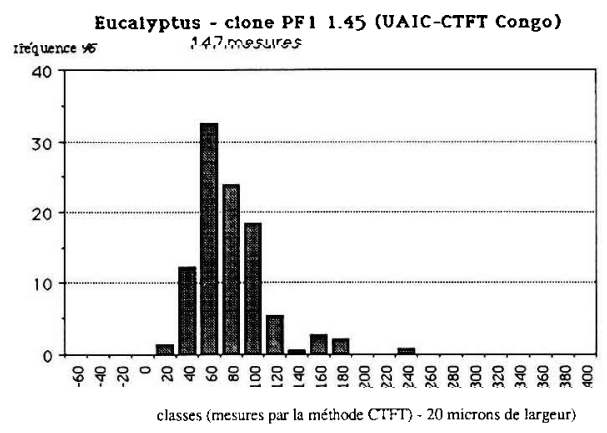

Fig 11. Distribution des déformations mesurées à la surface des eucalyptus.

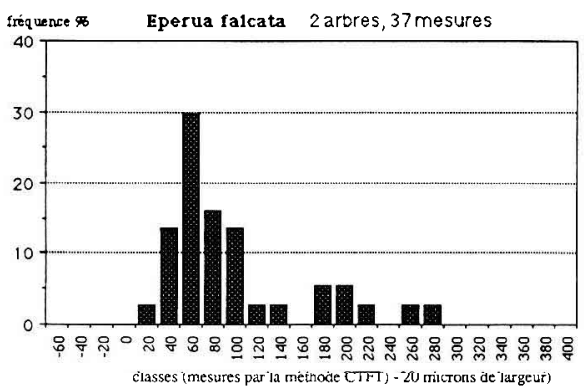

Fig 13. Distribution des déformations mesurées à la surface des Eperua falcata.

leurs conséquences sur l'interprétation des caractéristiques du bois dans l'arbre et de leur variabilité est donné par Chanson (1992) ; le présent compte rendu se limitera à rappeler et présenter les différents cas observés.

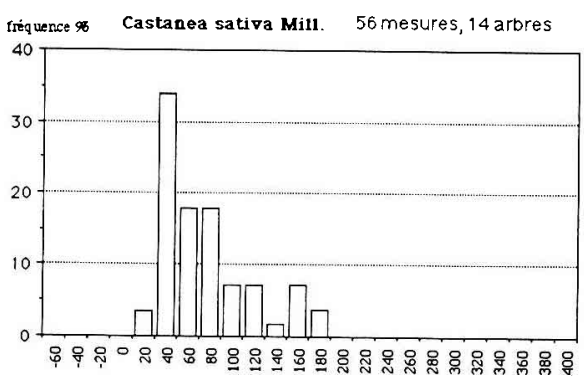

Fig 10. Distribution des déformations mesurées à la surface des Châtaigniers.

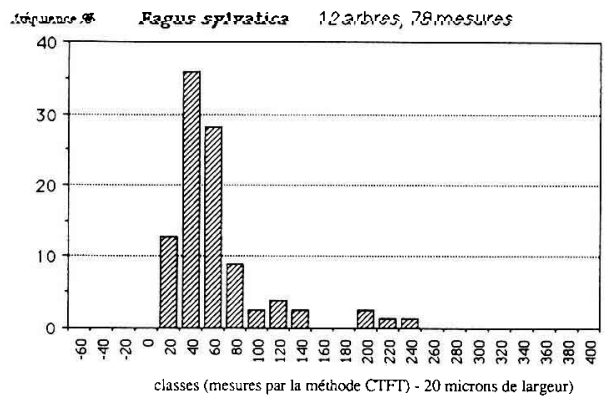

Fig 12. Distribution des déformations mesurées à la surface des hêtres.

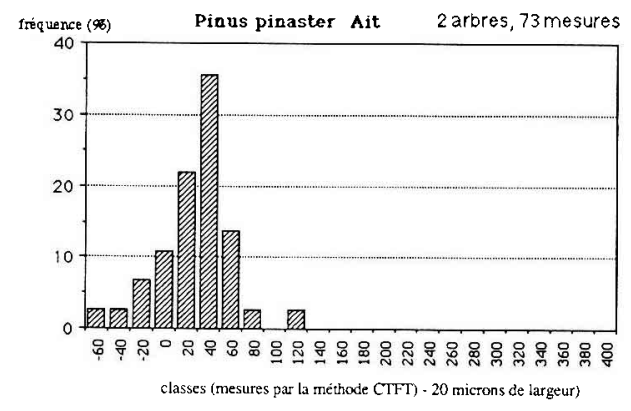

Fig 14. Distribution des déformations mesurées à la surface des Pins maritimes.

\section{Cas d'un pin maritime}

Dans le cas d'un avortement d'apex avec prise de relais d'une branche du dernier verticille formé, on observe une coulée de bois de compression sur la face inférieure de 
A)
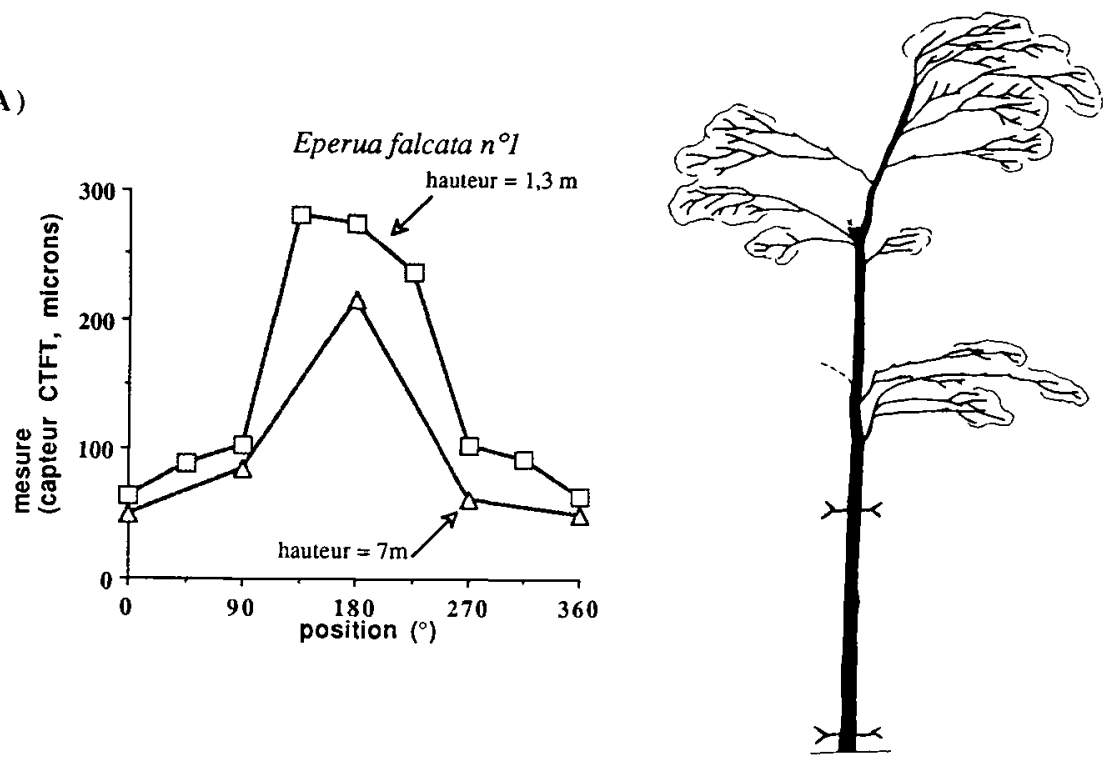

B)
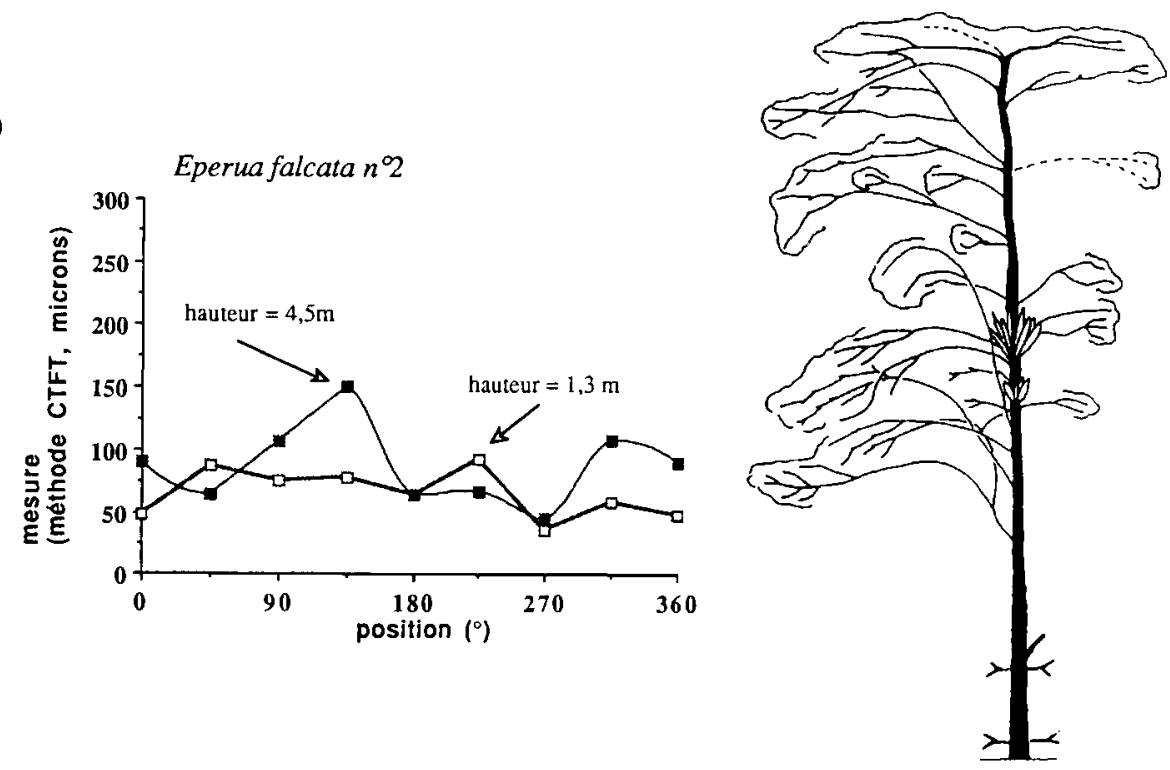

Fig 15. Morphologie de l'arbre et variations angulaires des déformations longitudinales chez 2 Eperua falcata. A. Pic important et unique sur un arbre incliné. B. Profil plus plat mais plus complexe sur un arbre droit présentant un grand nombre de "gourmands". 
A.
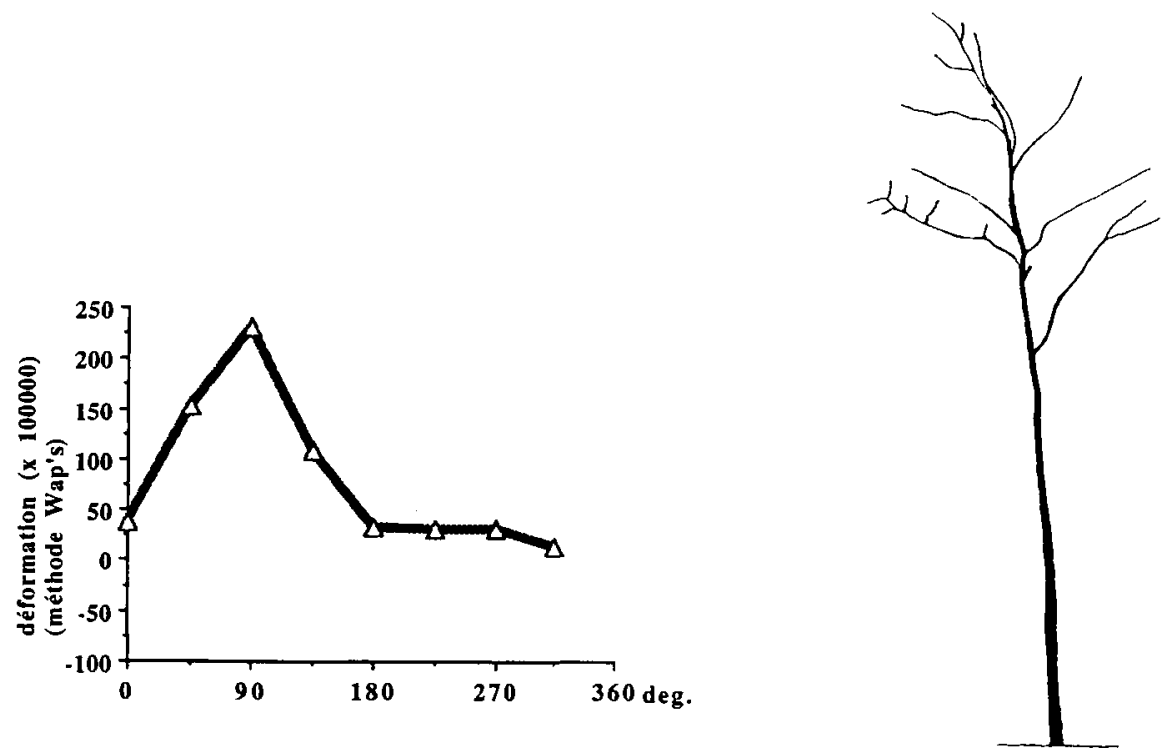

B.
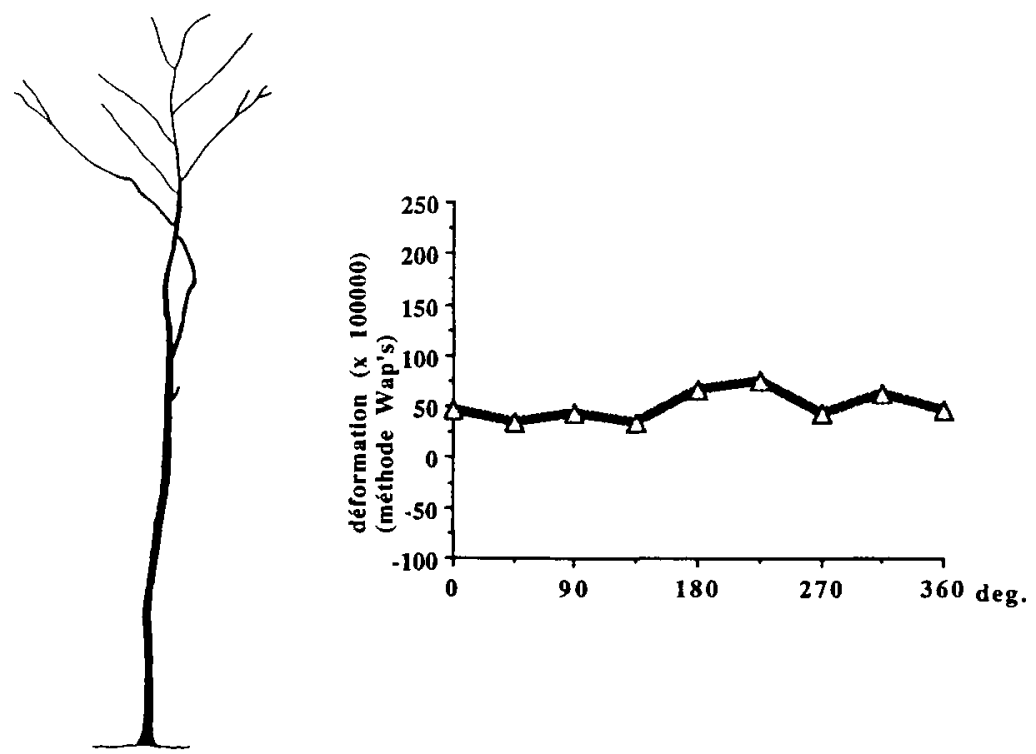

Fig 16. Morphologie de l'arbre et variations angulaires des déformations longitudinales chez 3 hêtres. A. Cas d'un arbre incliné. B. Cas d'un arbre droit. C. Cas d'un arbre présentant une grosse branche. 

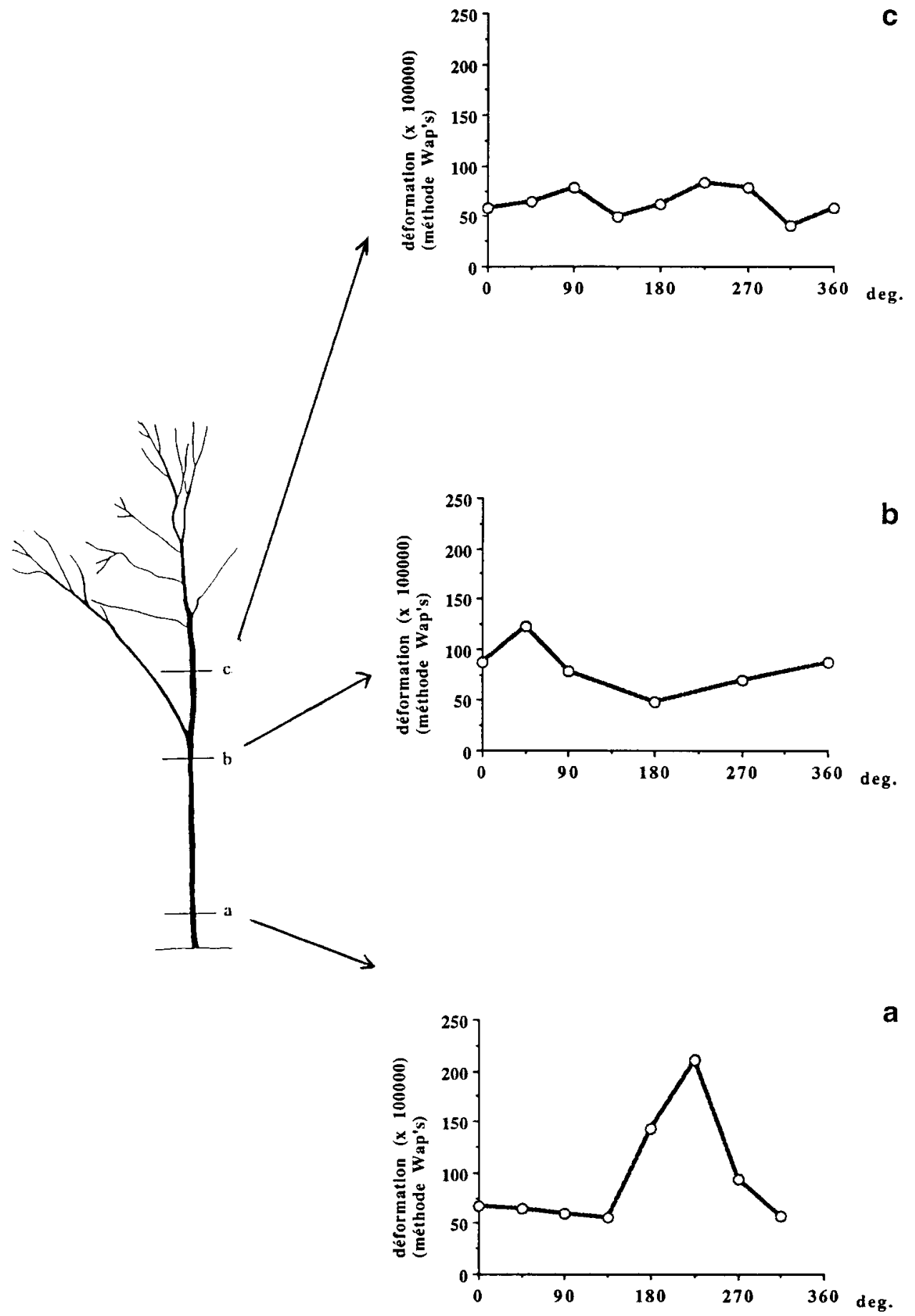
l'axe relais ainsi que du point de départ de la ramification jusqu'à la base du tronc (Loup et al, 1991).

\section{Cas d'un eucalyptus (clone 1.45)}

Ces clones, sélectionnés pour leur croissance rapide et leur rectitude, sont plantés dans un milieu extrêmement homogène. Morphologiquement, ils sont très proches: même dimension de couronne, formation de grosses branches non élaguées (réitérations) aux même niveaux, à la hauteur de $13 \mathrm{~m}$ puis de $22 \mathrm{~m}$. De fortes variations de la valeur (moyenne sur la circonférence) des déformations de surface avec la hauteur ont été observées, avec des ruptures de pente spectaculaires au passage des grosses branches (Baillères et al, 1992). Ce résultat reste pour l'instant délicat à interpréter.

\section{Cas des hêtres (Chanson et al, 1992 et fig 16)}

Les 2 premiers individus sont conformes aux cas typiques "droit» (profil plat) et "penché» (pic sur la face supérieure). En revanche, le troisième, bien que relativement droit, montre un pic important à l'opposé de la grosse branche qui déséquilibre le houppier. De fait, ce pic disparaît lorsqu'on passe cette branche en montant dans l'arbre.

\section{Observation de doubles pics}

Deux pics opposés sur la circonférence ont été observés sur Eperua falcata (fig 15B), Sacoglottis gabonensis (fig 17), Dichostemma sp (fig 18) et Castanea sativa (fig 19). L'interprétation fonctionnelle d'une telle distribution n'est pas immédiate puisque la conséquence n'est pas alors une réorien- tation de l'axe mais plutôt une tendance pour le tronc à "se couper en 2 ", chaque face «tirant" de son côté. De fait, ces distributions ont été observées sur des arbres sympodiaux (ie où le tronc est fait d'un empilement de relais) de sorte que ces doubles pics peuvent être interprétés comme un conflit entre les informations venant de l'ancien et du nouveau leader. Des études physiologiques conjointes seraient utiles pour approfondir ces hypothèses.

\section{CONCLUSION}

L'estimation des contraintes de croissance dans les arbres sur pied passe par la mesure de déformations résiduelles à la surface. Ce problème de la qualification d'un champ initial de contraintes internes n'est pas une mesure mécanique usuelle. En l'absence de norme établie, les différentes méthodes actuellement en cours sont susceptibles de donner des résultats parfois bien corrélés, parfois divergents puisque ces diverses méthodes font appel à des bases de mesure et des techniques très différentes. II serait donc utile de pouvoir s'appuyer sur une méthode de référence permettant au non spécialiste d'effectuer et de comparer des mesures. En l'état des connaissances, il nous semble que le capteur unidirectionnel mis au point au Centre technique forestier tropical, fondé sur la méthode du trou unique développée par Archer (1986a et b), serait l'outil le plus fiable et le plus aisément diffusable.

La variabilité des contraintes de croissance au sein d'une population est essentiellement liée aux variations intra-arbre, voire aux variations sur une même circonférence. L'apparition de pics angulaires de déformations liés à la formation de bois plus tendu (angiospermes) ou comprimé (gymnospermes) entraîne une non normalité des 


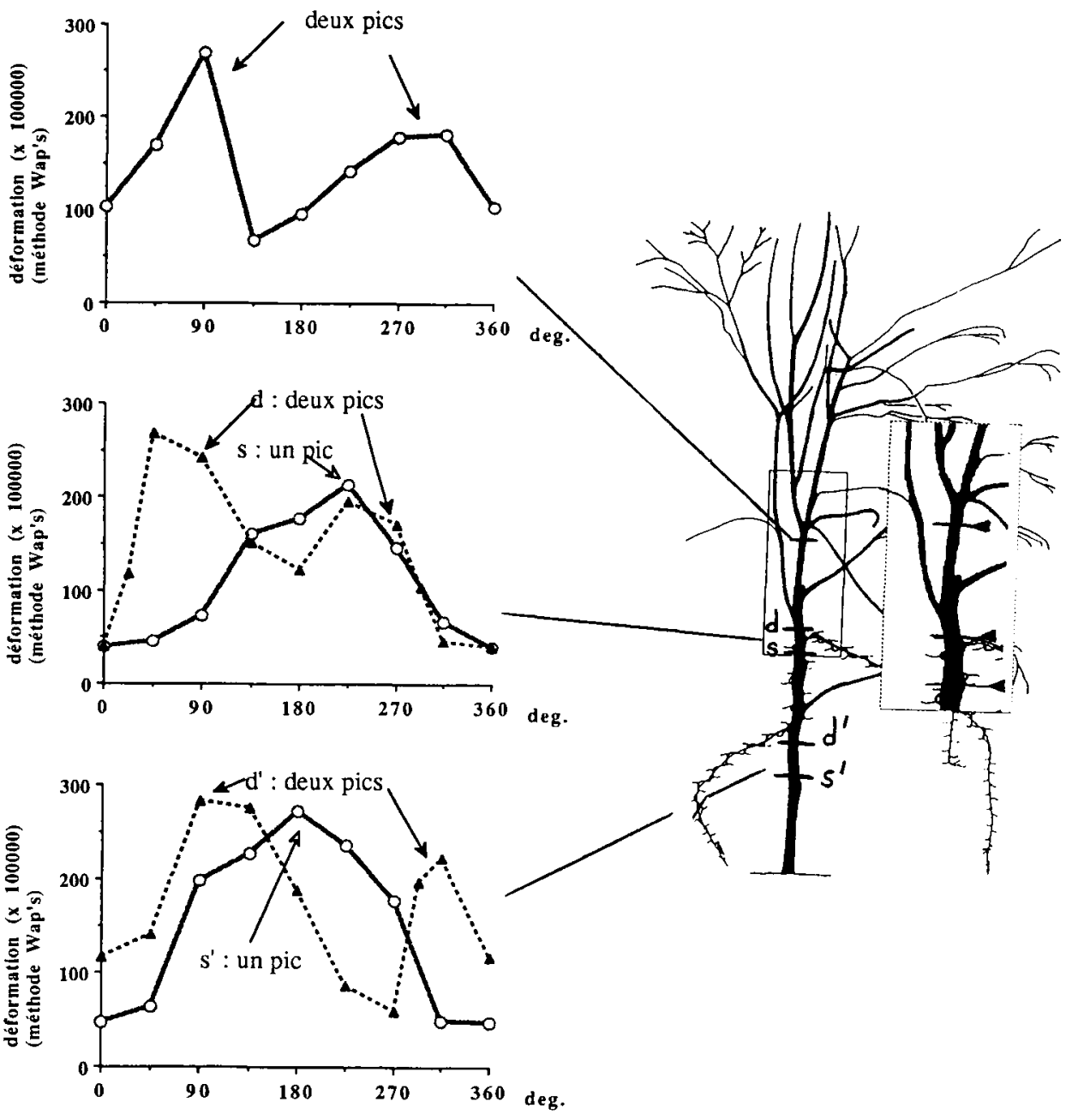

Fig 17. Observations de 2 pics de déformations longitudinales sous les insertions des branches relais chez un jeune Sacoglottis gabonensis.

valeurs mesurées. De plus, la différence entre 2 populations réside plus dans des différences de fréquences et d'amplitude de ces valeurs extrêmes que dans des différences entre les valeurs «normales» les plus fréquentes. D'un point de vue pratique, pour classer et qualifier des populations par rapport à leurs contraintes de croissance, il est donc intéressant d'effectuer un grand nombre de mesures par arbre et par circonférence, puis de qualifier les pics, plutôt que de raisonner sur des valeurs moyennes.

La mesure de déformations résiduelles à la surface des arbres sur pied permet d'accéder à une propriété rhéologique du bois qui vient juste d'être formé. Les variations des déformations mesurées sur la circon- 

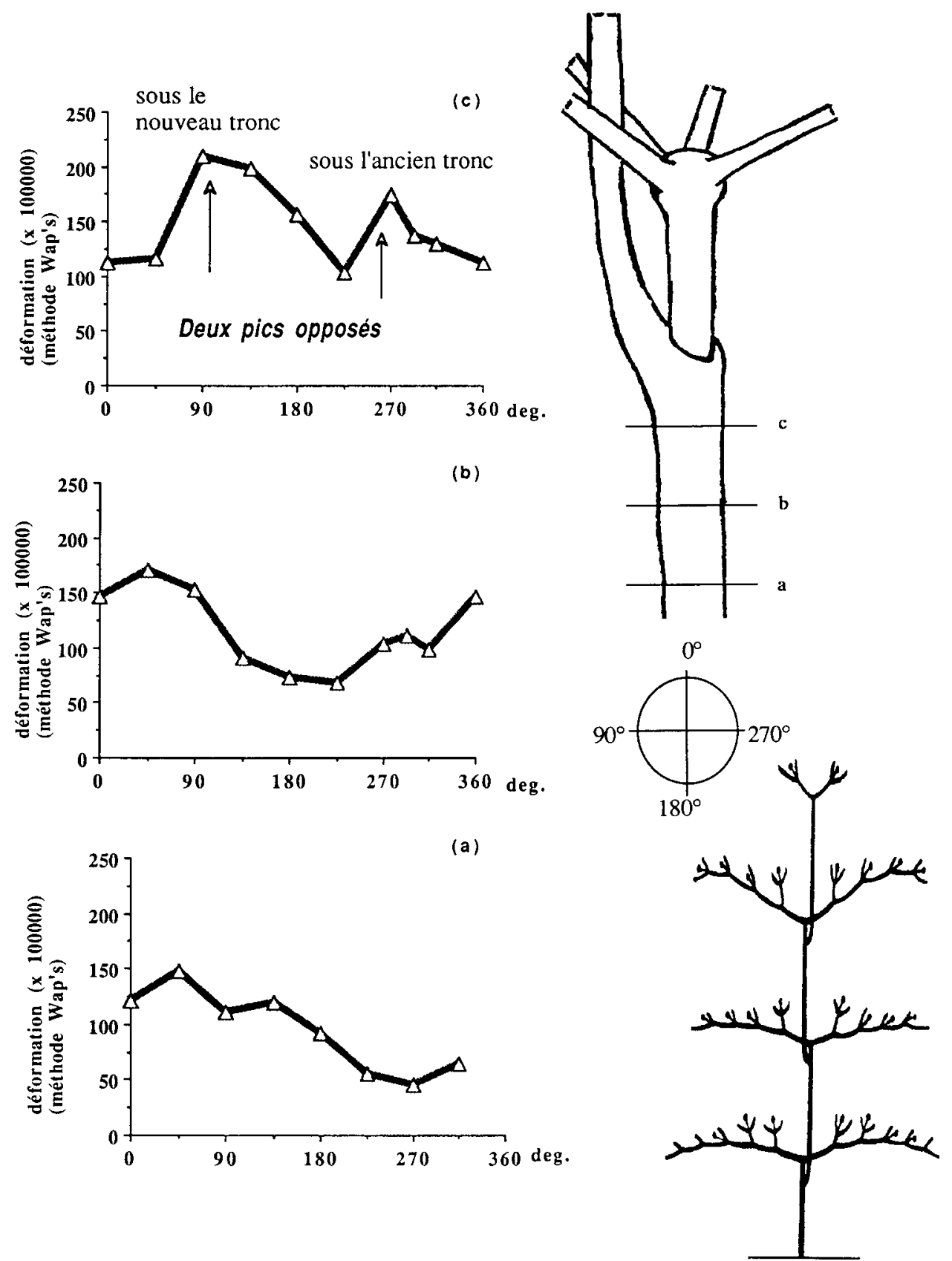

Fig 18. Observations de 2 pics de déformations longitudinales sous les insertions des branches relais chez Dichostemma sp. 


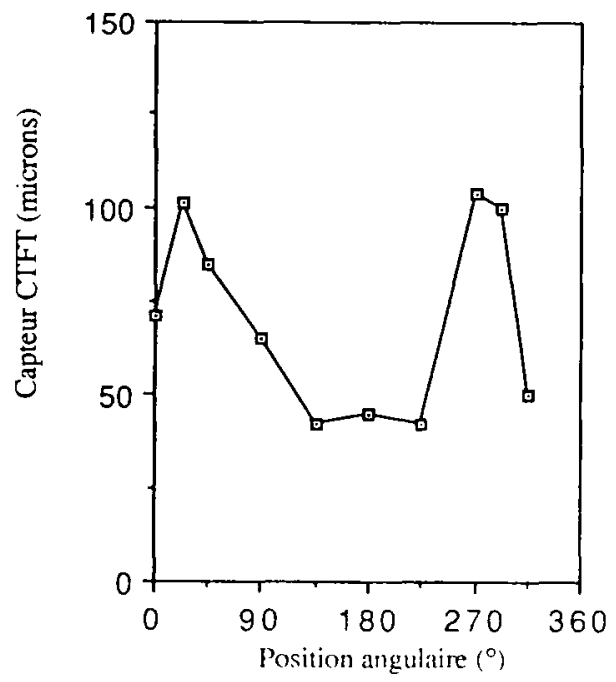

Fig 19. Observations de 2 pics de déformations longitudinales (section à $1,3 \mathrm{~m}$ ) chez Castanea sativa Mill.

férence et selon la hauteur semblent liées, d'une part, à des stratégies différentes de redressement ou plus généralement de réorientation des tiges (formation d'un "pic» dont la fonction est de courber la tige), d'autre part, à une organisation générale de l'arbre et aux différentes informations qui conditionnent le fonctionnement cambial. Les observations effectuées reliant déformations mesurées et morphologie des arbres ouvrent un large champ d'investigations tant pour la physiologie de l'arbre que pour les sciences du bois.

\section{REMERCIEMENTS}

Nous tenons à remercier collectivement tous les chercheurs du programme ASMA qui ont contribué à "engranger" les données exposées ici, et tout particulièrement $\mathrm{H}$ Baillères, $\mathrm{P}$ Castéra, JF Dhôte, J Gérard et C Loup.

\section{RÉFÉRENCES}

Archer RR (1986a) Growth stresses and strains. Springer Verlag,

Archer RR (1986b) Application of a new method for growth stress measurement to Pinus caribaea. Proc IUFRO Proj Group P5,01. Properties and utilization of tropical woods, Manaus, Brazil (19-23/11/84)

Baillères $\mathrm{H}$, Loup, Fournier $\mathrm{M}$, Chanson $B$, Gérard $J(1992)$ Contraintes de maturation en fonction de la hauteur et de l'âge chez de jeunes Eucalyptus de plantation. $4^{\mathrm{e}}$ séminaire "Architecture, Structure, Mécanique de l'arbre" (B Thibaut, ed) LMGC, Univ Montpellier II

Castéra P, Nepveu G, Mahé F, Valentin G (1994) Relationships between growth stresses, tension wood and felling shakes in poplar (Populus euramericana cv 1214). Ann Sci For (sous presse)

Chanson B (1993) Déformations de maturation: hétérogénéités angulaires en fonction du plan d'organisation des arbres. Séminaire du groupe d'étude de l'arbre, Paris 2 et 3 avril 1992, "Cambium, Production de bois et développement de l'arbre". Acta Bot Gallica140, 4, 395-401

Chanson B, Dhote JF, Fournier M, Loup C (1992) Dynamique des contraintes de croissance dans le bois de hêtre sur pied, en liaison avec la morphologie de l'arbre et l'expansion du houppier. Convention ONF-INRA n ${ }^{\circ}$ 12-90/03, rapport intermédiaire (mars 1992)

Fournier M (1989) Mécanique de l'arbre sur pied: maturation, poids propre, contraintes climatiques dans la tige standard. Thèse Doct INPL

Fournier M, Chanson B, Thibaut B, Guitard D. (1991) Mécanique de l'arbre sur pied : modélisation d'une structure en croissance soumise à des chargements permanents et évolutifs. II. Analyse tridimensionnelle des contraintes de maturation - cas du feuillu standard. Ann Sci For 48, 527-546

Gérard J, Garnier E, Florenchie P, Fournier M (1991) Déformations de sciages d'eucalyptus liées aux contraintes de maturation. $3^{e}$ Séminaire "Architecture, Structure, Mécanique de l'arbre" (B Thibaut, ed) LMGC, Univ Montpellier II

Guitard D (1987) Mécanique du matériau bois et composites. Cépadues-Editions, Collection Nabla, Toulouse 
Kübler H (1987) Growth stresses and related wood properties. Forestry abstracts Vol $48 \mathrm{n}^{\circ} 3$

Loup C, Fournier M, Chanson B (1991) Relations entre architecture, mécanique et anatomie de l'arbre : cas d'un pin maritime (Pinus pinaster Ait). L'arbre, biologie et développement. Naturalia monspelliensia ${ }^{\circ}$ hs (C Edelin, ed)

Poige $H$ (1981) Influence des éclaircies sur les contraintes de croissance du hêtre. Ann Sci For $38,407-423$
Saurat J, Gueneau P (1976) Growth stress in Beech. Wood Science \& Technology 10, 111 123

Serres D, Zarka J (1977) Sur une simulation numérique de la découpe des troncs d'arbres. Compte rendu de fin d'études DGRST, LMS, École Polytechnique, Palaiseau, France

Yamamoto, Okuyama T (1988) Analysis of the Generation Process of Growth stresses in cell walls. Mokuzai Gakkaishi 34, 788-793 